\title{
A New Terminal Nonsense Mutation of the Cathepsin C Gene in a Patient With Atypical Papillon-Lefèvre Syndrome
}

Moura $\mathrm{AL}^{1, *}$, Regateiro $\mathrm{FS}^{1,2, *}$, Peres Resende $\mathrm{E}^{3}$, Coimbra Silva $\mathrm{H}^{3}$, Gonçalo $\mathrm{M}^{4}$, Todo Bom $\mathrm{A}^{1,5}$, Faria $\mathrm{E}^{1}$

${ }^{I}$ Serviço de Imunoalergologia, Centro Hospitalar Universitário de Coimbra, Coimbra, Portugal

${ }^{2}$ Instituto de Imunologia, Faculdade de Medicina, Universidade de Coimbra, Coimbra, Portugal

${ }^{3}$ Instituto de Genética Médica, ICBR, Faculdade de Medicina, Universidade de Coimbra, Coimbra, Portugal

${ }^{4}$ Serviço de Dermatologia, Centro Hospitalar Universitário de Coimbra, Coimbra, Portugal

${ }^{5}$ Instituto de Fisiopatologia, Faculdade de Medicina, Universidade de Coimbra, Coimbra, Portugal

*Both authors contributed equally to this work.

J Investig Allergol Clin Immunol 2020; Vol. 30(2): 151-153 doi: $10.18176 /$ jiaci.0467

Key words: Atypical Papillon-Lefèvre syndrome. Cathepsin C. CTSC mutation. Papillon-Lefèvre syndrome. Immunodeficiency.

Palabras clave: Síndrome de Papillon-Lefèvre atípico. Catepsina C. Mutación CTSC. Síndrome de Papillon-Lefèvre. Inmunodeficiencia.

Papillon-Lefèvre syndrome (PLS, OMIM 245000) is a rare autosomal recessive disorder caused by mutations in the CTSC gene, which is located at 11q14.2 and encodes the enzyme cathepsin $\mathrm{C}$ [1]. Cathepsin $\mathrm{C}$ activates proinflammatory proteases (eg, NE, PR3, CatG, NSP4, chymases, tryptases, and granzymes), regulates the function of immune cells [2-4], and controls the formation of the corneocyte envelope.

PLS is characterized by symmetric palmoplantar keratoderma and severe, early-onset periodontitis, leading to the premature loss of dentition [1]. Other manifestations include recurrent pyogenic skin infections, nail dystrophy, hyperhidrosis, liver abscesses, mild mental retardation, intracranial calcifications, and increased susceptibility to infection [1,5]. CTSC loss of function results in reduced neutrophil response bacteria [2,3]. PLS is found in all ethnic groups, with an estimated prevalence of 1-4 per million individuals and a male:female ratio of 1:1 [1].

More than 75 biallelic hypomorphic variants in the CTSC gene have been described in PLS, Haim-Munk Syndrome (OMIM 245010), and aggressive periodontitis 1 (OMIM 170650) [6]. Most of the pathogenic variants causing PLS are located in exons 5-7, which encode the heavy chain of cathepsin $\mathrm{C}$, suggesting that tetramerization is important for enzymatic activity [7]. Given the high allelic heterogeneity, most patients are compound heterozygotes, with the exception of those from consanguineous families. Identical mutations of the CTSC gene can result in various phenotypes, and no straight genotype-phenotype correlations have been established $[7,8]$. Variations in intrafamilial penetrance have been described [7]. 
We report the case of a 26-year-old white man born to consanguineous Portuguese parents (coefficient of consanguinity $[\mathrm{r}], 1 / 32$ ) who developed the first symptoms at the age of 20 months. These comprised persistent gingivitis, palmoplantar skin thickening, periungual desquamation, recurrent episodes of furunculosis (buttocks, knees, and arms), and severe maxillary rhinosinusitis.

Dental caries were evident in the deciduous teeth, and only mild symptoms of periodontitis were observed during adolescence, with no loss of dentition. Oral and radiographic examinations at age 26 years showed preserved dentition with mild localized chronic periodontitis (less than $30 \%$ of the tooth and probing pocket depth of 4 to $5 \mathrm{~mm}$ ).

During adolescence, the patient continued to have mild palmoplantar keratoderma (trangrediens pattern) with erythema, psoriasiform plaques, and hyperhidrosis. At this age, recurrent skin abscesses and exuberant furunculosis necessitated several courses of antibiotics and prophylaxis, although these provided no benefit.

The skin infections worsened significantly at age 21 years, with several skin abscesses in the axillae and groin areas needing surgical drainage. The response to oral rifampicin and nasal mupirocin was transient. Bacterial swabs from the abscesses were positive for Staphylococcus aureus.

At the age of 25 years, the patient experienced a severe episode of pneumonia with several isolations of $S$ aureus and Klebsiella pneumonia in sputum. He required prolonged hospitalization. A computed tomography scan of the chest 4 months after the pneumonia episode showed cavitation suggesting pneumatoceles and left hilar lymphadenopathies (Figure).

The patient is currently receiving prophylaxis with oral cotrimoxazole and annual flu vaccination but continues to require an average of 3 courses of antibiotics and 1 surgical abscess drainage per year. Other manifestations include seborrheic dermatitis, allergic rhinitis, and lactose intolerance. Both parents and an older brother are healthy, and there is no reported family history of recurrent infections.

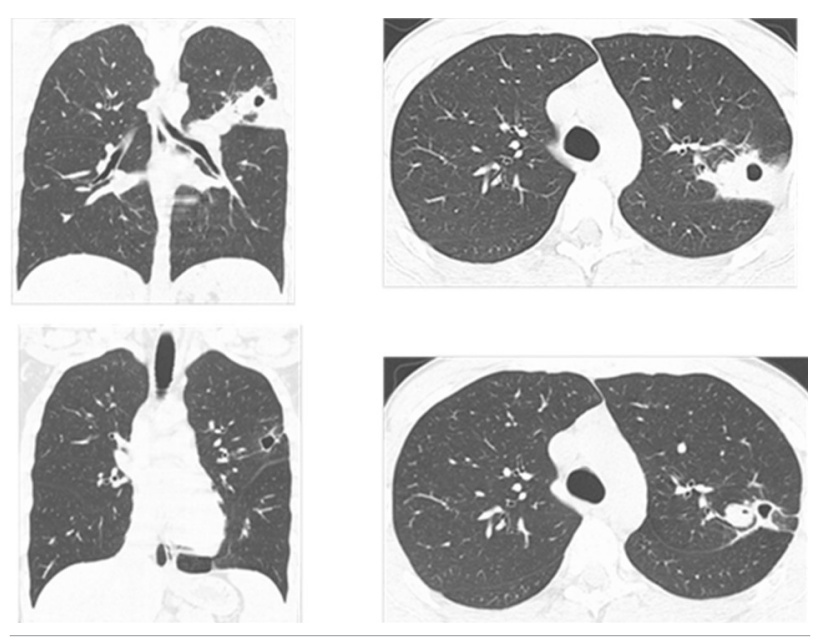

Figure. Images from a computed tomography scan of the chest during the pneumonia episode (upper row) and 4 months afer resolution (lower row), showing cavitation, pneumatoceles, and left hilar lymphadenopathies.
Full blood counts and values for immunoglobulin classes and subclasses were unremarkable, except for IgE (213 IU/mL). Skin prick tests were positive for Dermatophagoides pteronyssinus. Testing for human immunodeficiency virus was negative.

Flow cytometry of peripheral blood showed normal cell counts and percentages, as well as normal lymphocyte activation phenotypes. Furthermore, expression of CD40 in B cells was normal, as was expression of CD119, adhesion molecules CD11a/CD18, and CD54 on leukocytes. The expression of CD62L was low in lymphocytes and neutrophils. The result of the neutrophil oxidative burst assay was normal.

Sanger sequencing of the CTSC gene identified a novel homozygous nonsense variant in exon 7 (NM_001814.5:c.1339G>T; NP_001805.3: p.Glu447Ter) located in a conserved sequence ( 7 species, Ensembl), leading to a premature stop codon predicted to cause a terminally truncated protein. The variant is not on ClinVar (accessed May 7, 2019) but is referenced in the single-nucleotide polymorphism database (rs569702051) and described in ExAC with a frequency of $0.001 \%$ (http://exac.broadinstitute. org/gene/ENSG00000109861, accessed May 7, 2019). Both parents were heterozygous for the variant. The patient's brother refused to undergo genetic testing.

The patient presented an atypical course of PLS, with notably mild hyperkeratosis and periodontitis, while showing increased susceptibility to severe infections.

The variant of CTSC present in this patient supports a clinical diagnosis of PLS. This newly described variant can be considered "likely pathogenic", as it is found in very low frequencies in the population and leads to a premature stop codon with truncation of a conserved region in the light-chain domain. While very terminal, the variant may interfere with the tetramerization of the mature enzyme. Importantly, another missense variant in the same residue-albeit compound heterozygous - was described in PLS (p.E447G) [5-7].

The reasons for the dissociation between mild hyperkeratosis and dental manifestations and the major infectious manifestations are unclear, although they may include particularities of the CTSC mutation or other gene-gene interactions and/or environmental factors. Despite an extensive immunological work-up, no other immunodeficiencies were found. The absence of significant immunological anomalies is consistent with previous reports, and more subtle functional defects may be present. Reduced cathepsin C enzymatic activity results in diminished activity and stability of neutrophil-derived serine proteases [9], such as elastase and cathepsin $\mathrm{G}$, which are thought to play a role in the regulation of innate immune responses to bacteria. However, neutrophils in PLS do not uniformly have a defect in their ability to kill bacteria, suggesting that serine proteases do not represent the major mechanism used by human neutrophils for killing common bacteria [3]. Other roles in neutrophil function have also been described $[9,10]$.

In PLS, NK cells contain inactive granzyme B and have a cytolytic defect, failing to induce the caspase cascade in target cells [4]. However, in vitro activation of NK cells with interleukin 2 in PLS restores cytolytic function and granzyme $\mathrm{B}$ activity by means of a cathepsin $\mathrm{C}$-independent mechanism [4]. PLS patients were recently shown to have 
impaired autophagy caused by a CTCS mutation and insufficient lysosomal function [10].

The increasing number of atypical PLS cases reported, including those with isolated keratosis or periodontitis, highlights the importance of correct diagnosis. Early diagnosis is beneficial for treatment and long-term quality of life.

\section{Funding}

The authors declare that no funding was received for the present study.

\section{Conflicts of Interest}

Dr. Todo Bom reports grants and personal fees from Novartis, Mundipharma, GlaxoSmithKline, Teva Pharma, Sanofi, and AstraZeneca and grants from Leti outside the submitted work.

The remaining authors declare that they have no conflicts of interest.

\section{References}

1. Dhanrajani PJ. Papillon-Lefevre syndrome: clinical presentation and a brief review. Oral Surg Oral Med Oral Pathol Oral Radiol Endod. 2009 Jul;108(1):e1-7.

2. Korkmaz B, Caughey GH, Chapple I, Gauthier F, Hirschfeld J, Jenne $D E$, et al. Therapeutic targeting of cathepsin C: from pathophysiology to treatment. Pharmacol Ther. 2018 Oct;190:202-36

3. Pham CT, Ivanovich JL, Raptis SZ, Zehnbauer B, Ley TJ. PapillonLefevre Syndrome: Correlating the Molecular, Cellular, and Clinical Consequences of Cathepsin C/Dipeptidyl Peptidase I Deficiency in Humans. J Immunol. 2004 Dec 15;173(12):727781.

4. Meade JL, De Wynter EA, Brett P, Sharif SM, Woods CG, Markham AF, et al. A family with Papillon-Lefèvre syndrome reveals a requirement for cathepsin $C$ in granzyme $B$ activation and NK cell cytolytic activity. Blood. 2006 May 1;107(9):36658.

5. Hart PS, Zhang Y, Firatli E, Uygur C, Lotfazar M, Michalec MD, et al. Identification of cathepsin C mutations in ethnically diverse papillon-Lefèvre syndrome patients. J Med Genet. 2000 Dec;37(12):927-32

6. Hart TC. Localisation of a gene for prepubertal periodontitis to chromosome 11q14 and identification of a cathepsin $C$ gene mutation. J Med Genet. 2000 Feb;37(2):95-101.

7. Nagy N, Vályi P, Csoma Z, Sulák A, Tripolszki K, Farkas K, et al. CTSC and Papillon-Lefèvre syndrome: detection of recurrent mutations in Hungarian patients, a review of published variants and database update. Mol Genet Genomic Med. 2014 May;2(3):217-28.

8. Noack B, Görgens $H$, Schacher B, Puklo $M$, Eickholz $P$, Hoffmann $T$, et al. Functional Cathepsin $C$ mutations cause different Papillon-Lefèvre syndrome phenotypes. J Clin Periodontol. 2008 Apr;35(4):311-6.

9. Guarino C, Hamon Y, Croix C, Lamort AS, Dallet-Choisy $S$, Marchand-Adam $S$, et al. Prolonged pharmacological inhibition of cathepsin $C$ results in elimination of neutrophil serine proteases. Biochem Pharmacol. 2017 May 1;131:5267.

10. Bullón P, Castejón-Vega B, Román-Malo L, Jimenez-Guerrero MP, Cotán D, Forbes-Hernandez TY, et al. Autophagic dysfunction in patients with Papillon-Lefèvre syndrome is restored by recombinant cathepsin C treatment. J Allergy Clin Immunol. 2018 Oct;142(4):1131-43.e7.

Manuscript received July 23 2019; accepted for publication November 20, 2019.

Emília Faria

Serviço de Imunoalergologia Centro Hospitalar e Universitário de Coimbra Praceta Prof. Mota Pinto 3000-075 Coimbra, Portugal E-mail: emiliafaria@gmail.com 